\title{
Cuidadoras inmigrantes: trabajo emocional
}

\author{
Inmigrants caregivers: emotional labor
}

\section{Imigrantes Cuidadoras: trabalho emocional}

\author{
Monserrat Ruiz López ${ }^{1}$; Manuel Moreno Preciado² \\ ${ }^{1}$ Profesora asociada. Universidad Europea de Madrid. \\ ${ }^{2}$ Profesor Titular. Universidad Europea de Madrid \\ Cómo citar este artículo en edición digital: Ruiz López, M. y Moreno Preciado M. (2013) \\ Cuidadoras inmigrantes: trabajo emocional. 17, 35. Disponibleen: <http://dx.doi.org/10.7184/cuid.2013.35.08> \\ Correspondencia: Montserrat Ruiz López ProfesoraAsociada de laUniversidadEuropea de Madrid. Departamento de Enfermería. \\ Villaviciosa de Odón. Dirección C/ Tajo, s/n. Urb. El Bosque. 28670 Madrid. \\ Correo electrónico: montserrat.ruiz@uem.es \\ Recibido: 09/12/2012. Aceptado: 21/02/2013
}

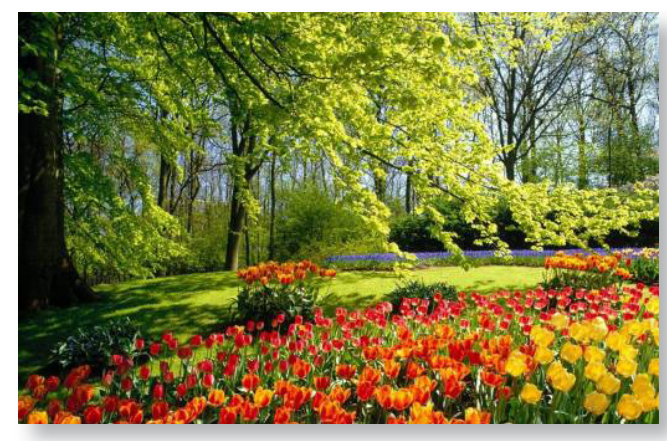

\section{ABSTRACT}

The changes in demographic, economic, socio-cultural and health foster the emergence of a new set of caregivers: the hired caregiver, covered generally by immigrant women. This article is a study that aims to inquire into this new reality, focusing mainly on knowing the experiences and emotions of immigrant carers in relation to people and their environment maintained.

The study, a qualitative research, collected through direct testimony of caregivers, which is their lived experiences, that is, the stories of a difficult and complex context, where it crosses a double need for affection: on one hand, the one of those suffering bereavement of immigration, by the distancing of their own family, by the harshness and precariousness of their migrant status and on the other hand, those who in old age, beyond physical dependence, suffer from loneliness. It demonstrates the emotional and relational nature of care and the positive contributions of these immigrant women to a situation characteristic of global societies, and with aging populations.

Keywords: caregivers, immigrants, dependency, emotional care.

\section{RESUMO}

As mudanças de caráter demográfico, económico, socio-culturais e de cuidados de sáude, propiciam a aparição de uma nova figura de cuidadores: o cuidador contratado, posto de trabalho, em regra geral, ocupado por mulheres imigrantes. $\mathrm{O}$ presente artigo pretende indagar sobre esta nova realidade, procurando fundamentalmente conhecer as vivências e emoções das cuidadoras imigrantes e a sua relação com as pessoas a que cuidam e os meio envolvente.

O estudo de caráter qualitativo, recolhe através de testemunhos diretos das cuidadoras, o que constitui a sua experiência vivida; quer isto dizer, os relatos sobre um contexto difícil e complexo, donde se cruzam uma necessidade dupla de afeto: por um lado, as que sofrem um "luto migratório", pela separação dos seus 
famiiares, pela dureza e precariedade de sua condição de imigrantes, e por outro lado, a de quem na velhice, para além da dependência física, sofrem de solidão. Torna-se evidente o caráter afetivo e de relação do cuidado e as contribuições positivas destas mulheres imigrantes a uma situação caraterística das sociedades globais, e com populações envelhecidas.

Palavras-chave: cuidadoras, imigrantes, dependência, cuidados emocionais.

\section{RESUMEN}

Los cambios de carácter demográfico, económicos, socioculturales y sanitarios propician la emergencia de una nueva figura de cuidadores: el cuidador contratado, cubierto, en general, por mujeres inmigrantes. El presente artículo trata de un estudio que pretende indagar sobre esta nueva realidad, centrándose, fundamentalmente, en conocer las vivencias y emociones de las cuidadoras inmigrantes en su relación con las personas cuidadas y su entorno.

El estudio, de corte cualitativo,recoge a través de testimonios directos de las cuidadoras, lo que constituye su experiencia vivida; es decir, los relatos sobre un contexto difícil y complejo, donde se cruza una doble necesidad de afecto: de una parte, la de quienes sufren el duelo migratorio, por el alejamiento de los suyos, por la dureza y la precariedad de su condición de migrantes y por otra parte, la de quienes en la vejez, más allá de la dependencia física, sufren de soledad. Se evidencia el carácter afectivo y relacional del cuidado y las aportaciones positivas de estas mujeres inmigrantes a una situación característica de las sociedades globales, y con poblaciones envejecidas.

Palabras clave: cuidadoras, inmigrantes, dependencia, cuidados emocionales.

\section{INTRODUCCIÓN}

La Ley de Promoción de la Autonomía Personal yAtención a las personas en situación de Dependencia (cuarto pilar del estado del bienestar), seis años después de su aprobación no ha conseguido dar respuesta a las expectativas y necesidades de las personas dependientes y familias. Entre las personas mayores se concentra el mayor número de personas en situación de discapacidad y limitación de la autonomía. El aumento de la esperanza de vida, junto con la baja natalidad ha hecho de España uno de los países más envejecidos. Según el informe sobre la población mayor, elaborado por el Instituto de Mayores y Servicios Sociales (IMSERSO, 2010), España se sitúa a la cabeza de los países europeos enesperanza de vida, dato sólo superado por Suecia y Francia. La esperanza de vida en las mujeres se sitúa en 84 años y en los hombres en los 78 años, y la tendencia según este mismo organismo continuará en aumento. Se prevé que en el 2030, la esperanza de vida en las mujeres alcanzará los 86,9 años y en los varones subirá hasta los 80,9 años.

De otra parte, debido a los avances de las ciencias biomédicas y a la mejora de las condiciones de vida, ha aumentado la supervivencia de las personas con enfermedades crónicas, trastornos degenerativos o con secuelas de accidentes de tráfico o laborales. Esto provoca, como dice Ma Ángeles Durán (2006), una desproporción entre la necesidad o demanda de cuidados y los servicios disponibles.

El modelo de familia española que, tradicionalmente, venía siendo un gran soporte de cuidados para sus seres queridos está cambiando; la sustitución de la familia extensa por la familia nuclear con pocos hijos, así como la aparición de nuevas formas de unión como la familia monoparental hace que disminuyan 
en los hogares el número de cuidadores informales disponibles. Esto, unido a la mayor movilidad geográfica de las personas, conlleva que ya no se comparta el mismo techo con la generación de los abuelos, como en épocas anteriores. No obstante, todavía es importante el número de personas dependientes a cargo de sus familias (Panizo Robles, 2007), las cuales consideran que las instituciones no pueden suplir el afecto de la familia. De hecho, las personas mayores en su mayoría prefieren vivir en sus hogares (IMSERSO, 2010).

La incorporación de la mujer al mundo laboral, acompañado de la ausencia de políticas que compatibilicen la vida familiar y laboral, es otro factor clave en este cambio de modelo y el elemento que con mayor fuerza explica el déficit de cuidadores. Produciéndose lo que los autores denominan "crisis del sistema informal de cuidados" (Robledo Martin et al., 2008; Galiana-Gomez, et al., 2008); la cual está siendo paliada, en parte, por la emergencia de la nueva figura del "cuidador-contratado". Son, por lo general, mujeres inmigrantes que prestan cuidados y atención, fundamentalmente en el domicilio, pero también en entornos institucionalizados.

Los cuidados que prestan las mujeres inmigrantes a las personas dependientes, se han convertido en una ayuda fundamental para las familias.Como apuntan Berjano y Lujan: "El cuidado prestado por mujeres inmigrantes se convierte a veces en el único recurso de las familias a la par que es una excelente oportunidad para la inserción laboral de estas mujeres. El cuidado realizado por estas personas es una importante aportación en el cuidado a la dependencia, emergiendo nuevos actores en el sistema de atención a la salud” (2004:125).Esta figura del "inmigrante cuidador" tiene una imagen social más positiva que la que habitualmente transmite el discurso mediático pues visualiza la contribución de los inmigrantes en la solución de determinados problemas sociales (Moreno Preciado, 2008).

Aunque, comúnmente, la limpieza del hogar y el cuidado de las personas se engloban dentro de la denominación "trabajo doméstico" se trata, en realidad,de actividades muy diferentes ya que, mientras que en lo primero predominan las tareas mecánicas, en lo segundo se precisa de una relación cara a cara. Autoras como Horshschild reconocen el carácter emocional del cuidado: "Con el término 'cuidar' me refiero a un vínculo emocional, usualmente reciproco, entre la persona que brinda el cuidado y la que la recibe. En el marco de ese vínculo, la persona que brinda el cuidado se siente responsable por el bienestar de otros y lleva a cabo un trabajo mental, emocional y físico a fin de cumplir con su responsabilidad. Por consiguiente, cuidar una persona implica interesarse por ella” (2008:309). Una conceptualización muy parecida elabora Marie FrançoiseCollière (1993) para quien el cuidado se entiende como todas aquellas actividades humanas físicas, mentales y emocionales dirigidas a mantener la salud y el bienestar del individuo y/o la comunidad.

Cuidar es un acto (o conjunto de actividades) no siempre medibles, ya que la calidad del mismo, depende del vínculo emocional que se establece persona a persona, más allá del carácter contractual o voluntario del mismo. También desde las ciencias sociales, se destaca el carácter emocional del cuidado. En este sentido, Susan Himmelweit (2004) describe las características del cuidado, haciendo énfasis en el componente relacional y emotivo. (Ver cuadro 1).

En el estudio del IMSERSO (2005) sobre cuidadoras inmigrantes, se evidencia la difusa 
relación (o incluso confusión) entre la parte laboral y la afectiva que éstas mantienen con las personas que cuidan. Más recientemente, desde las Ciencias de la Salud, diversos estudios consultados, informan de los vínculos afectivos que se establecen con la persona cuidada y la mujer inmigrante (Galiana-Gómez et. al., 2008; Alegre Ruiz de Mendoza, 2011; Serrano Molina et al. 2011).

El cuidado en el ámbito doméstico es algo que permanece oculto (DomínguezAlcón, 1986) como actividad propia de la mujer; entendemos que en mayor grado ese ocultamiento se produce cuando se realiza en las condiciones de precariedad y subordinación de las cuidadoras inmigrantes-contratadas. Nos preguntamos ¿cómo se desarrolla esa actividad? ¿Qué vínculos se tejen entre cuidadoras y personas dependientes? ¿Qué valor añadido aportan a la función de cuidar? El propósito de este estudio es favorecer que emerjan esas voces silenciadas. En este artículo nos centraremos, principalmente, en los factores emocionales y afectivos del cuidado.

\section{OBJETIVOS}

\section{Objetivo general:}

- Conocer las vivencias y percepciones de las cuidadorasinmigrantes y sus aportaciones en el cuidado, así como los vínculos afectivos y emocionales que se tejen con las personas dependientes.

\section{Objetivos específicos:}

- Conocer detalladamente el tipo de actividades que desarrollan.

- Favorecer la expresión de sentimientos en relación a las personas cuidadas.

- Favorecer la expresión de sus necesidades afectivas en relación a sus condiciones sociolaborales y emocionales.

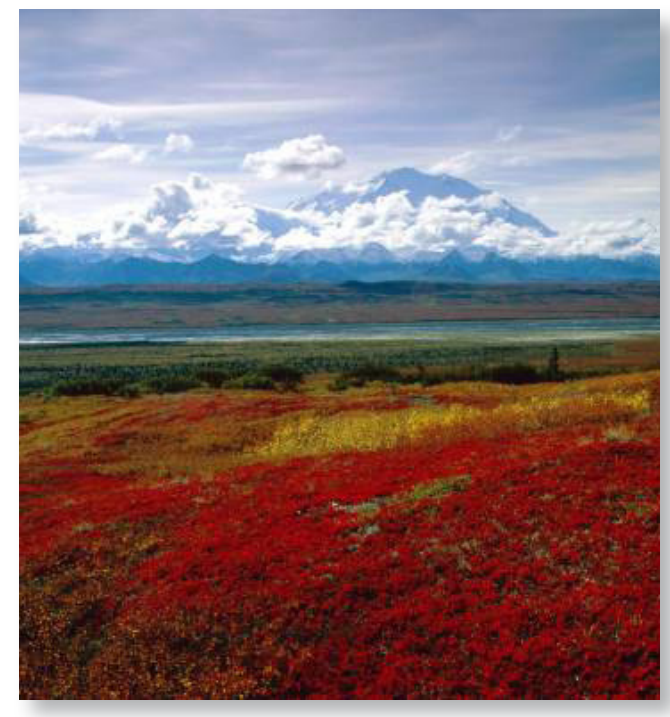

\section{METODOLOGÍA}

El estudio es una investigación cualitativa con metodología fenomenológica y hermenéutica la cual nos permite aproximarnos al fenómeno objeto de estudio. El método fenomenológico busca descubrir los fenómenos experimentados por las personas a través del análisis de sus descripciones; la hermenéutica entiende que los humanos experimentan el mundo a través del lenguaje (Solano, 2006) y esto, a su vez, nos permite comprender a las personas y sus experiencias vividas.

La selección de informantes se realizó a través de dos ONGs de la Comunidad de Madrid, entre las participantes a cursos de cuidados básicos organizados por dichas organizaciones. Se admitió a cuantas personas aceptaron ser entrevistadas.

La principal técnica de recogida de datos fuela entrevista en profundidad. Siguiendo la lógica de la investigación cualitativa entendemos las entrevistas como flexibles, dinámicas y dialógicas, como una conversación entre iguales y por tanto como "no directivas, no estructuradas, no estandarizadas y abiertas" (Taylor y Bogdan, 2008). 
Para la producción de los datos se realizaron veinte entrevistas (Cuadro 2), entre los meses de noviembre 2011 a mayo 2012. Las entrevistas tuvieron lugar en la misma sede donde tenían lugar los cursos de formación. Fueron grabadas y transcritas literalmente.

Para el análisis de los datos hemos utilizado el método de las comparaciones constantes propuesto por Glaser y Strauss (1967), que consiste en generar teoría fundamentada en estudios enmarcados dentro del paradigma fenomenológico. Este método sirve de guía durante el proceso analítico en estudios cualitativos favoreciendo la conceptualización de los datos de manera inductiva (Carrillo Pineda et al., 2011).

El análisis se llevó a cabo en tres niveles delimitados como sugiere el método de las comparaciones constante (Strauss y Corbin, 2002). En el primer nivel o de "codificación abierta" de carácter descriptivo se intentó identificar categorías de significado. En un segundo nivel o de "codificación axial" se buscan las relaciones coherentes entre las categorías y se agrupan en otras unidades más amplias, denominadas metacategorias. Finalmente, en un tercer nivel de análisis "codificación selectiva" se identifica una categoría central o "vector cualitativo". (Ver cuadro 3).

Esta investigación se ha desarrollado en el respeto de los derechos de los informantes, desde su participación voluntaria, la plena información sobre los objetivos del estudio, así como la garantía del anonimato (los nombres que aparecen son figurados).

\section{RESULTADOS}

Partiendo del análisis de los datos empíricos que recogen la perspectiva de los informantes sobre su experiencia vivida presentaremos a continuación los principales resultados. (Ver cuadro 3)

\section{El contexto}

\section{"Chicas para todo"}

En ningún caso existe su trabajo se limita al cuidado si no que se les contrata también para las actividades domésticas clásicas como limpiar, cocinar, hacer la compra, etc. Complementan, suplen y supervisan el cuidado de los mayores a su cargo, ayudándoles también en la administraciónde los tratamientos prescritos por su médico. Les vigilan cuando existen trastornos cognitivos, les llevan al médico y administran cuidados técnicos cuando su estado empeora.

- "Limpiaba, los atendía, hacia la compra, la lavadora. Les hacia compañía" (Ana).

- "No es difícil manejar la medicación por tú te haces un record de las que le das todos los días. La médica iba todos los martes y revisaba las anotaciones de la tensión arterial, del azúcar. La médica era de Sanitas revisaba y me lleva unas hojas que eran como las del hospital. Cuando vino el problema, cuando empezaron las escaras, cuando le dio el tercer Ictus" (Carolina).

\section{Tiempo y espacio en el cuidado}

Cuando el trabajo se realiza en condiciones de precariedad, como es el caso de las cuidadoras, es muy difícil guiar la relación laboral por los criterios tradicionales donde se delimita y separa el ámbito laboral del personal y la duración de la prestación del servicio. Esta frontera queda totalmente diluida cuando se trata de cuidadoras internas:

- "Yo estoy interna y es verdad que no tengo mucho que hacer pero tengo una responsabilidad porque le pasa algo a esa señora y la responsabilidad es mía porque yo estoy ahí para cuidar esa señora en el tiempo que estoy contratada" (Elena).

La gran responsabilidad y el estar pendiente todo el día y en ocasiones la noche, requiere 
presencia y dedicación completa durante las veinticuatro horas del día:

- "Vigilar constantemente a la persona que tu estas cuidando, tratar de darle la comida a tiempo a su hora, darle la medicación a su hora, mantenerlo limpio, aseado porque todo eso entra dentro de los cuidados. Hacerle un poquito de gimnasia, caminar. Estar vigilante a todos los intereses que tiene esa persona. Mantener limpio donde se acuesta, en donde orina, mantener el baño limpio" (Carolina).

- "Hago la casa y estoy pendiente todo el día porque siempre me está llamando porque tiene retención de orina y cada 5 minutos o 2 o $3 \mathrm{mi}$ nutos me llama para llevarla al baño. Ella me toca el timbre y yo estoy haciendo una cosa y tengo que ir [...] cada vez que voy al baño estoy como unos 20 minutos" (Alba).

\section{Duelo migratorio}

Al difícil contexto sociolaboral se añade el inevitable duelo por lo que se ha dejado atrás. Las cuidadoras no pueden desprenderse de la pena y el desgarro que supone la lejanía de los suyos. La responsabilidad y el sentimiento de culpa afloran de forma permanente:

- "Esto es terrible. Yo y mis almohadas son los que saben cuantas noches llorando" (Natalia).

- "El problema de estar interna no es el trabajo porque yo decía que den trabajo todo el día y así no tengo que pensar en mi familia" (Rocío).

\section{Lo laboral y lo afectivo: límites difusos}

\section{Ser humano: animal narrativo}

Las largas jornadas de trabajo, sobre todo en las cuidadoras internas, favorecen la creación de sólidos lazos de relación con las personas dependientes. En muchas ocasiones y por diferentes causas, sus familiares no les prestan suficiente atención. Estas mujeres les demues- tran su cariño en muchos hechos del día a día, en cómo les echan de menos cuando no están con ellos o como continúan apreciándolos incluso habiendo dejado de ser sus cuidadoras. Su cercanía a estas personas las hace receptoras de sus necesidades comunicativas y afectivas:

- "Les gusta a ellos que les hables, que les acompañes. Que les hables, que ellos también te cuenten sus cosas y que estés pendientes de ellos. Quieres merendar, quieres un vasito de agua. Cuando yo llegaba decía como me desespero porque llegues porque no sé con quién hablar" (Cristina).

- "Los hijos no tienen tiempo con las cosas del día a día, muchas cosas, están acá para allá y no tienen tiempo para los mayores. Y como nosotros estamos cuidándolos yo entiendo que me toca a mi tener ese cariño" (Julia).

- "La señora no quería comer sola. La señora era charlatana y le gustaba charlar, me contaba cómo era la juventud, cómo era la guerra civil todo eso, me contaba y yo le decía bueno todo eso ha pasado también en mi país, yo también le contaba como es mi país como es mi familia todo" (Maria).

\section{"El roce hace el cariño":}

A través del contacto directo se van tejiendo lazos de afectividad donde y aparecen las muestras de agradecimiento propias de la hospitalidad:

- "No sé se te van metiendo poco a poco porque al fin y al cabo yo no sabía que quería tanto a la abuela hasta que me iba, la tenía en la cabeza y decía Dios mío estará bien, me mortificaba y yo loca por regresarme porque pensaba que me la iba a encontrar muerta" (Nuria).

Una cuidadora, llegó a un domicilio tras estar en un trabajo donde había sufrido una importante discriminación, después de esta experiencia comenzó a cuidar de una perso- 
na parapléjica y de su marido. Estuvo con ellos durante 14 años:

- "Pero yo le digo cuando llegué a la casa donde estuve ellos no fueron mis jefes, fueron mis padres, ambos. Como ellos eran así uno no tenía cansancio para hacerles nada, incluso creo que me faltó tiempo para hacer más cosas por ellos, porque ellos se merecen eso y mucho más" (Natalia).

Para otra cuidadora la persona que atendió y que luego falleció le ayudó en todos los trámites para legalizar su situación y poder traer a su hijo para que lo operaran en Madrid:

- "No te gusta que aparezca una persona nueva a cuidarla, porque entonces como se iba a sentir, yo por lo menos era de confianza. Yo le dije que hasta el último día la iba a cuidar y la cuide. En ese momento no tienes valor para decir me voy. Dejas incluso de cuidar tu vida. Trabajo me ofrecieron mucho en aquel entonces" (Irene).

\section{La complejidad del cuidado}

\section{Vigilancia en el cuidado}

Las cuidadoras se preocupan por el bienestar de la persona que tienen a su cargo. Se sienten responsables de la vida de la persona mayor. Están muy atentas a la administración de la medicación y a los brotes agudos de las enfermedades:

- "Yo estoy interna y es verdad que no tengo mucho que hacer pero tengo una responsabilidad porque le pasa algo a esa señora y la responsabilidad es mía porque yo estoy ahí para cuidar esa señora en el tiempo que estoy contratada" (Elena).

- "Tienes que estar muy atenta si no quieres que le pase algo. Si eres un poco responsable muy atenta" (Begoña).

Una de las cuidadoras para supervisar a la mujer que estaba cuidando, cuando ésta enfer- maba se acostaba con ella en la misma cama:

- "Me preocupa por eso cuando ella está enferma pongo más cuidado y si puedo me duermo con ella en la misma cama. Porque no quiero que pase nada y además yo tengo todos los medicamentos que ella toma” (Julia).

\section{Sin alivio del peso del cuidador}

Es un trabajo para el que se requiere mucha paciencia no sólo por las largas jornadas sino, también, por el carácter de las personas mayores. Las cuidadoras refieren el enorme desgaste físico y psicológico de su trabajo.

- "Paciencia, mucha paciencia, paciencia y paciencia. Hay veces que te dicen cosas que no te hubiera gustado oír, pero al ser mayores no es que estén locos, pero ellos no coordinan. A veces te dicen cosas que no te quieren decir y si tu lo tomas a pecho pues vas de trabajo en trabajo" (Lidia).

- "Mucha paciencia. Paciencia y respeto también. Por ejemplo con la persona que estoy ahora, a veces tiene malos días y te chilla. Tu no puedes levantarle la voz cuando se le pase el enfado hablas con ella y le dices mira esto no me gusto" (Irene).

Se sienten cansadas por las jornadas continuas sin descanso pero lo que realmente les resulta agotador es cuando no pueden dormir:

- "No puedo decir las horas que trabajo porque ya una de ellas está muy desvalida y a la medianoche te llama y dice que quiero agua o que quiero levantarme. Yo no podría decir cuántas horas trabajo. Hay noches que no duermo pero bueno ese es mi trabajo. Por lo menos una o dos noches a la semana no duermo" (Monica).

Cuando las personas se encuentran en estados avanzados de demencia es frecuente que la cuidadora se instale en la misma habitación para poder ejercer mejor el control de la persona: 
- "Estoy sola todo el día y toda la noche, con el tercer ictus fue cuando me toco dormir con él. $\mathrm{Tu}$ sabes que cuando ellos tienen estos problemas no distinguen tiempo, estaba todo el tiempo con la barandilla tic-tac y no me dejaba dormir" (Carolina).

El cansancio hace que aparezcan trastornos de la salud en el cuidador:

- "Cuando no podía dormir, cuando empezó la demencia que comenzaba por las noches a chillar, a decir socorro ladrones y se quería tirar de la cama. Nosotras dormíamos en dos camitas en la misma habitación. [...] y yo de los nervios se me caían las cosas, me dolía la cabeza y lo llevaba fatal" (Nuria).

- "Tuve que ir directamente a un psiquiatra. Tuve una depresión que engorde 14 kilos. Me pasaba la noche sin dormir y claro a comer chocolate y helados. Cogí bulimia nerviosa. Te destroza totalmente. Y tu venga a comer porque qué otra cosa vas a hacer... última hora no dormía ni de día ni de noche" (Irene).

\section{CONCLUSIONES}

1. El estudio evidencia la esencia afectiva y emotiva del cuidado.

2. En el caso de las cuidadoras inmigrantes este vínculo afectivo viene motivado por dos razones principales. La primera, tiene que ver el contexto en el cual se realiza su actividad laboral: un ámbito delimitado, cerrado y en torno a personas en situación, no sólo de dependencia física y psíquica, sino también de soledad y falta de afectividad. La segunda, se refiere a las condiciones del proceso migratorio donde en su fase central (primeros años de estancia en el país) aparece con fuerza el duelo migratorio (Achotegui, 2010), que comporta necesidad de afectividad y la posibilidad de expresar emociones. Se trata del encuentro entre dos estados de vulnerabilidad.
3. Los condicionantes de una relación contractual caracterizada por la precariedad que, en buena lógica, invitarían a limitar el trabajo a las prestaciones establecidas, se ven superadas por la existencia de un vacío afectivo que sólo su presencia puede rellenar.

4. El tiempo y el espacio. La procedencia de las mujeres cuidadoras contratadas de países con una estructura familiar tradicional favorece su inserción al ámbito doméstico y al cuidado de las personas dependientes. Las diferencias culturales, lejos de constituir una barrera en la relación con las personas dependientes parecen, por el contrario, un elemento enriquecedor; aportan habilidades y conocimientos, fruto de la experiencia adquirida en el ámbito doméstico y familiar de sus países de origen, que se concreta en todo un elenco de cualidades esenciales del cuidado como son la paciencia y dedicación para las situaciones difíciles y también la necesaria capacidad de observación y vigilancia.

5. Cuidar de una persona es algo totalmente distinto de mantener un hogar. Las actividades prácticas de carácter doméstico pueden realizarse sin la mediación de otra persona; $\sin$ embargo, para cuidar es necesario que se establezca un vínculo relacional. Apoyándonos en estas premisas, entendemos que cuidar personas desde el sistema informal supone aportar confort, ayudar o suplir en actividades que la persona no pueda realizar por ella misma, ayudar en el seguimiento de los tratamientos del sistema formal, acompañarles al médico, realizar gestiones administrativas, coger el teléfono, salir de paseo, ser nexo de unión entre la persona y su entorno más cercano, en definitiva acompañar.

6. Se evidencia que las mujeres cuidadoras contratadas constituyen un complemento ideal (en algunos casos, más que complemen- 
tar son un sustituto) para las familias ya que no sólo cubren actividades domésticas y cuidadoras sino que también establecen una relación basada en las emociones.

\section{BIBLIOGRAFÍA}

- Achotegui, J. (2010) Emigrar en el siglo XXI. El Síndrome de Ulises. Síndrome del inmigrante con estrés crónico múltiple. El mundo de la mente, Figueras.

- Alegre Ruiz de Mendoza, A. (2011) Cuidar en una cultura diferente: vivencias de cuidadoras de origen latinoamericano en el país vasco. Index de Enfermería 20 (1-2), 21-25.

- BerjanoPeirats, E. y Lujan López, E. (2004) Inmigración y cuidados a mayores. "Un mercado no tan invisible". Rev. Geriatrika 20(3), 124-132.

- Carrillo Pineda, M., Leyva-Moral, J.M. y Medina, JL. (2011)El análisis de los datos cualitativos: un proceso complejo.Index de Enfermería (20), 96-100.

- Collière, Ma F. (1993) Promover la vida. McGraw-Hill Interamericana, Madrid.

- Domínguez Alcón, C. (1986) Los cuidados y la profesión enfermera, Pirámide, Madrid.

- Duran, Ma A. (2006) Dependientes y cuidadores: el desafío de los próximos años. Revista del Ministerio de Trabajo y Asuntos Sociales 60, 57-73.

- Galiana-Gómez, Ma J., De la Cuesta-Benjumea, C. y Donet-Montagut, T. (2008) Cuidadoras inmigrantes: características del cuidado que prestan a la dependencia. EnfermeriaClínica, 18 (5), 269-272.

- Glaser, B., Strauss, A. (1967) Discovery of grounded theory. Aldine, Chicago

- Himmelweit, S. (2004) La economía de la atención. En Congreso Internacional SARE 2003. Ekamunde, San Sebastián. Disponible en: http://www.emakunde.euskadi. net/u72-publicac/es/contenidos/informacion/pub_jornadas/es_emakunde/adjuntos/sare2003_es.pdf

- Hoschschild, A. (2008) La mercantilización de la vida íntima. Katz editores, Madrid.

- Instituto de Mayores y Servicios Sociales (2005) Cuidados a la dependencia e inmigración. Informe de resultados. IMSERSO, Madrid.
- (2010). Encuesta de mayores 2010. Disponible en:http:// www.imserso.es/InterPresent2/groups/imserso/documents/binario/presentacionencuestamayores_20.pdf

-Moreno Preciado, M. (2008) El cuidado del “otro". Bellaterra, Barcelona.

- Panizo Robles, J.A. (2007) La cobertura social de la dependencia (con ocasión de la Ley 39/2006, de 14 de diciembre, de promoción de la autonomía personal y atención a las personas en situación de dependencia). Revista de Trabajo y Seguridad Social del Centro de Estudios Financieros, 286, 49-106.

- Robledo Martin, J., Martin -Crespo, Ma C. y Salamanca Castro, A.B. (2009) Factores socioculturales determinantes de desigualdades en salud en inmigrantes latinoamericanas cuidadoras de ancianos, XIII Encuentro Internacional de Investigación en Enfermería, Alicante.

- Serrano Molina, L., Nieto Blanco, E., MorianoBéjar, P., Vicente del Hoyo, M., Montero Martin, S., Moreno Torrecillas, E. y Dávila Álvarez, V. (2011) Análisis de las necesidades y expectativas de pacientes, cuidadores familiares y cuidadores contratados en el servicio de asistencia domiciliaria en Atención Primaria. Enfermería Comunitaria, 7(1).Disponible en: http://www.index-f. com/comunitaria/v7n1/ec7287.php

- Solano, Ma C. (2006) Fenomenología- Hermenéutica y Enfermería. Cultura de los cuidados, 19, p. 5.

- Taylor, S.J. y Bogdan, R. (2008) Introducción a los métodos cualitativos en investigación. Paidós, Barcelona.

- Strauss, A., Corbin, J. (2002) Bases de la investigación cualitativa. Técnicas y procedimientos para desarrollar la teoría fundamentada. Universidad de Antioquia, Bogotá. 


\section{CUADRO 1: características del cuidado (Himmelweit, 2004)}

- El cuidado constituye un servicio que ha de prestarse en el mismo momento que se consume.

- La necesidad de atención suele ser urgente por tanto ha de prestarse cuando y como sea necesario.

- La atención es un servicio personal; por lo que el producto de la atención no puede separarse de la persona que lo presta.

- La atención supone un trabajo emocional, es decir, se supone que las emociones de la persona que lo realizan afecta a la calidad del producto.

- La atención es algo más que una labor emocional transitoria, constituye el desarrollo de relaciones, así como la prestación de un servicio.

- Esto implica la importancia de la persona que realiza el trabajo de atención, las personas no pueden intercambiarse en dichas labores. Las personas aprenden a atender a otras en particular.

- Las habilidades implícitas en la atención son en gran parte, aunque no totalmente, específicas para un tipo de persona, desarrollándose este aprendizaje a través de la experiencia personal.

\begin{tabular}{|l|l|l|l|l|}
\hline $\begin{array}{l}\text { Nombre } \\
\text { figurado }\end{array}$ & Edad & Nacionalidad & $\begin{array}{l}\text { Nivel } \\
\text { sociocultural }\end{array}$ & $\begin{array}{l}\text { Interna/ } \\
\text { externa }\end{array}$ \\
\hline Alba & 38 & Bolivia & Medio & Interna \\
\hline Laura & 40 & Perú & Bajo & Interna \\
\hline Pilar & 47 & Perú & Bajo & Externa \\
\hline Paula & 38 & Ecuador & Bajo & Interna \\
\hline Miriam & 36 & Ecuador & Medio & Interna \\
\hline Ana & 27 & Perú & Medio & Interna \\
\hline Ana & 40 & Perú & & Interna \\
\hline Julia & 47 & Brasil & Alto & Interna \\
\hline Maria & 47 & Bolivia & Bajo & Interna \\
\hline Carolina & 54 & Colombia & Alto & Interna \\
\hline Margarita & 39 & Bolivia & Bajo & Interna \\
\hline Sonia & 57 & Colombia & Bajo & Interna \\
\hline Natalia & 47 & Ecuador & Bajo & Interna \\
\hline Nuria & 31 & $\begin{array}{l}\text { Santo } \\
\text { Domingo }\end{array}$ & Alto & Interna \\
\hline Irene & 29 & Cuba & Medio & Interna \\
\hline Cristina & 28 & Bolivia & Bajo & Interna \\
\hline Monica & 44 & Perú & Alto & Externa \\
\hline Lidia & 41 & Ecuador & Alto & Interna \\
\hline Lucia & 46 & Rumania & Alto & Externa \\
\hline Begoña & 38 & Bulgaria & Medio & Externa \\
\hline
\end{tabular}

CUADRO 2:

perfil de las personas del estudio

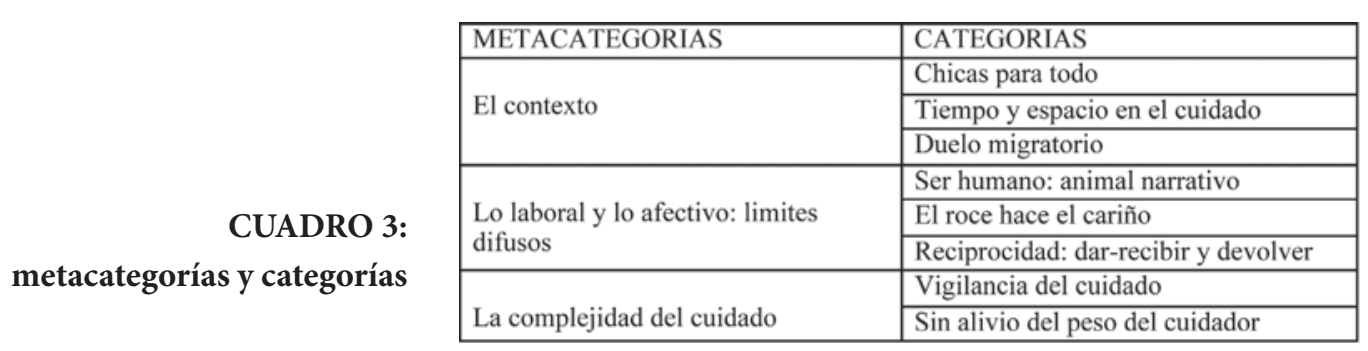

